\title{
Increased Tone of the Human Colon Muscle by Bisacodyl In Vitro
}

\author{
Yang Won Min, ${ }^{1}$ Eun-ju Ko, Jeong Hwan Kim, ${ }^{3}$ Ji Yeon Lee, ${ }^{4}$ Hee Cheol Kim, ${ }^{5}$ Woo Yong Lee, ${ }^{5}$ and Poong-Lyul Rhee ${ }^{1 *}$ \\ ${ }^{I}$ Department of Medicine, ${ }^{2}$ Biomedical Research Institute, and ${ }^{5}$ Department of Surgery, Samsung Medical Center, Sungkyunkwan University \\ School of Medicine, Seoul, Korea; ${ }^{3}$ Department of Internal Medicine, Digestive Disease Center, Konkuk University Medical Center, Konkuk \\ University School of Medicine, Seoul, Korea; and ${ }^{4}$ Department of Physiology, University of Nevada School of Medicine, Reno, Nevada, USA
}

\section{Background/Aims}

Although bisacodyl is a widely administered laxative, its underlying mechanism of action remains generally unknown. This study focuses on investigating the effects of bisacodyl on the human colon muscle contraction, and elucidating its mechanism of action.

\section{Methods}

Sigmoid colon muscle strips (20 longitudinal and 18 circular muscles) were obtained from 20 subjects who underwent colectomy for colon cancer. Isometric force measurements were calculated in response to electrical field stimulation (EFS, 0.3 milliseconds in trains of $10 \mathrm{~Hz}$ for 20 seconds, $150 \mathrm{~V}$ ). Peak and nadir (tone) during and after EFS, were measured in a controlled state, and after sequential addition of bisacodyl $(1 \mu \mathrm{M})$, atropine $(1 \mu \mathrm{M})$, N-nitro-L-arginine (L-NNA, $100 \mu \mathrm{M})$, MRS2500 $(1 \mu \mathrm{M})$, and tetrodotoxin $(\mathrm{TTX}, 1 \mu \mathrm{M})$ to the organ bath.

\section{Results}

Transient phasic contractions were observed during EFS, and after cessation of EFS. In the longitudinal muscles, nadir during EFS, and tone after EFS, significantly increased after addition of bisacodyl, and persisted after sequential addition of atropine, L-NNA, MRS2500, and TTX, indicating a direct action of bisacodyl on the smooth muscle. In the second experiment, pretreatment of TTX abolished EFS-induced phasic contractions. Although no phasic contraction was produced after perfusion of bisacodyl, tone was increased, thereby supporting evidence of a direct mechanism of action of bisacodyl on the colon smooth muscle.

\section{Conclusions}

Bisacodyl increases the tone of longitudinal muscle in the human sigmoid colon through a direct action on the smooth muscle. Further study is warranted to investigate the neural mechanism of action of bisacodyl.

(J Neurogastroenterol Motil 2018;24:317-323)

\section{Key Words}

Bisacodyl; Colon; Humans; Muscle contraction; Physiology

Received: May 18, 2017 Revised: September 25, 2017 Accepted: November 20, 2017

(a) This is an Open Access article distributed under the terms of the Creative Commons Attribution Non-Commercial License (http://creativecommons. org/licenses/by-nc/4.0) which permits unrestricted non-commercial use, distribution, and reproduction in any medium, provided the original work is properly cited.

*Correspondence: Poong-Lyul Rhee, MD, PhD Department of Medicine, Samsung Medical Center, Sungkyunkwan University School of Medicine, 81 Irwon-ro, Gangnam-gu, Seoul 06351, Korea Tel: +82-2-3410-3409, Fax: +82-2-3410-6983, E-mail: plrhee@skku.edu 


\section{Introduction}

Bisacodyl is a diphenylmethane stimulant laxative, which is frequently administered for treating functional constipation, bowel preparation before colonoscopy, and pharmacologic provocation during colon manometry. ${ }^{1-3}$ Bisacodyl induces high-amplitude propagating contractions (HAPCs), which is the colonic propulsive motor activity. ${ }^{3-5}$ However, the underlying mechanism of action of bisacodyl on the colon is largely unknown.

Guidelines for medical management of chronic constipation recommend bisacodyl as the second line treatment, when bulk or osmotic laxatives are ineffective, for improving bowel movement and stool consistency. ${ }^{1,6}$ Although it is unlikely that chronic use of bisacodyl is harmful to the colon, it could induce abdominal discomfort and cramping pain. ${ }^{7,8}$ In addition, new drugs such as lubiprostone, linaclotide, and prucalopride have been developed, and could be administered when conventional laxatives fail to manage constipation, and irritable bowel syndrome with constipation. ${ }^{9-12}$ Thus, bisacodyl needs to be efficiently administered with a consideration of its mechanism of action.

This study aims to investigate the effects of bisacodyl on the human colon muscle contraction and to elucidate its mechanism of action.

\section{Materials and Methods}

\section{Subjects and Tissues}

All patients provided written informed consent, before inclusion in this study. The study protocol was conducted in accordance with the Declaration of Helsinki and approved by the Institutional Review Board of Samsung Medical Center, Seoul, South Korea (No. 2012-02-031, 20th March 2012). Sigmoid colon tissues were collected from 20 subjects (14 males, median age 62 years, age range 50-77 years) who underwent colectomy for colon cancer at Samsung Medical Center from October 2016 to March 2017. Shortly after colectomy, colon smooth muscle specimens were collected from regions free of macroscopic evidence of cancer infiltration. The specimens were collected in cold oxygenated modified Krebs-Ringer bicarbonate solution $\left(97 \% \mathrm{O}_{2}\right.$ and $\left.3 \% \mathrm{CO}_{2}\right)$, and immediately transported to the laboratory. After specimens were pinned to the base of a Sylgard Silicone Elastomer dish, the mucosa was extracted via sharp dissection. Then, thin strips of tissues were cut in parallel to the muscle fibers in $2 \times 10 \mathrm{~mm}$ slices.

\section{Organ Chamber Experiment}

Experiments were performed in vitro with strips of both longitudinal $(n=20)$ and circular $(n=18)$ muscles. The mechanical activities were recorded, as changes in isometric force using standard organ bath techniques, previously described in another study conducted by our laboratory. ${ }^{13-16}$ Isolated muscle strips were attached to a fixed mount, and to a Fort 10 isometric strain gauge (UC3-GOULD Instruments, Paris, France; FT03-GFASS, Warwick, RI, USA). The strips were immersed in organ baths maintained at $37 \pm 0.5^{\circ} \mathrm{C}$ with oxygenated Krebs-Ringer bicarbonate solution. After stabilization for 60 minutes, electrical field stimulation (EFS) of intramural nerves was carried to attached strips by 2 platinum ring electrodes with an optimal frequency and duration ( 0.3 milliseconds in trains of $10 \mathrm{~Hz}$ for 20 seconds, $150 \mathrm{~V})$. The electrodes were connected to a GRASS S88 (GRASS Instruments, Quincy, MA, USA) stimulator.

\section{Experimental Protocol and Measurement}

We measured EFS-induced contractile responses in a control state and after the administering of bisacodyl ( $1 \mu \mathrm{M}$; Merck KGaA, Darmstadt, Germany). To understand the cholinergic, purinergic, and nitrergic roles in the EFS-induced response under bisacodyl perfusion, atropine (a muscarinic antagonist, $1 \mu \mathrm{M}$ ), Nnitro-L-arginine (L-NNA; a nitric oxide synthase inhibitor, 100 $\mu \mathrm{M}$ ), and MRS2500 (a purinergic $\mathrm{P}_{2} \mathrm{Y}_{1}$ antagonist, $1 \mu \mathrm{M}$ ), were added in a sequential order to the organ bath. Then, tetrodotoxin (TTX, $1 \mu \mathrm{M}$ ) was applied to inhibit any nerve-mediated contraction. Muscle strips (13 longitudinal and 12 circular muscles) were perfused with each drug for at least 20 minutes after EFS, and had a recovery time for 5 minutes before perfusion of the next drug (Fig. 1).

Peak (the highest value) and nadir (the lowest value) amplitudes of both circular and longitudinal muscle strips were measured with a passive tension of $0.7 \mathrm{~g}$ during EFS of 20 seconds. After cessation of EFS, post-stimulus rebound (PSR) responses were produced as reported in the previous study. ${ }^{17}$ Thus, we also measured peak and tone of PSR. PSR tone was defined as the value 2 minutes after the initiation of EFS. In addition, late (stabilized) tone was measured as the value just before perfusion of the next drug (Fig. 2).

To test the direct effect of bisacodyl on the colon smooth muscle, muscle strips (7 longitudinal and 6 circular muscles) were immersed in TTX-containing organ bath, and were stimulated before and after additional perfusion of bisacodyl (Fig. 3). 


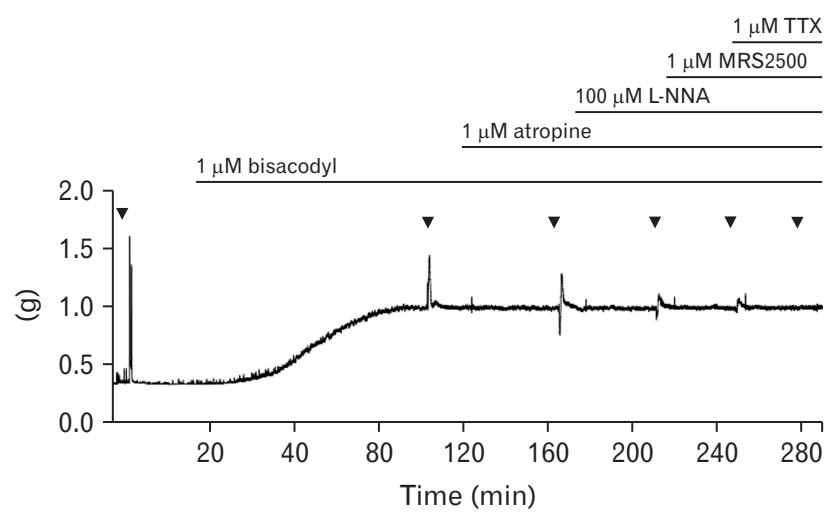

Figure 1. Typical trace of electrical field stimulation-induced responses of the human colon smooth muscle strip after serial administration of bisacodyl, atropine, N-nitro-L-arginine (L-NNA), MRS2500, and tetrodotoxin (TTX) in the longitudinal muscle.

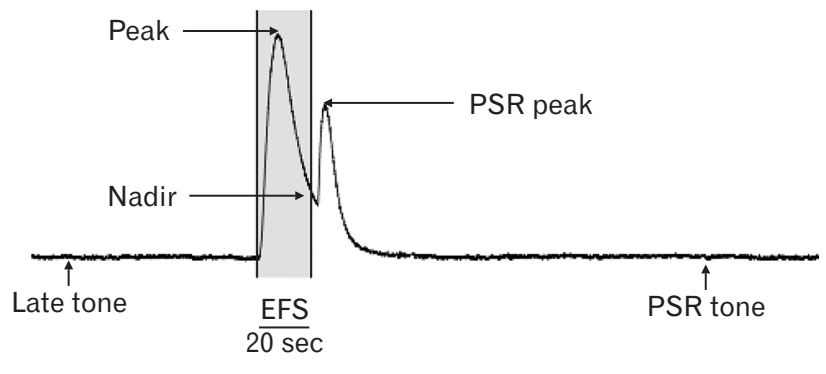

$2 \min$

Figure 2. Electrical field stimulation (EFS)-induced responses of the longitudinal colon muscle. Peak is the highest value, while nadir represents the lowest value during EFS of $20 \mathrm{~s}$. PSR (post-stimulus rebound) peak is the highest value during PSR response and PSR tone is the value 2 mins after the initiation of EFS. Late tone was defined as the value just before perfusion of the next drug.

\section{Statistical Methods}

Data are expressed as mean $\pm \mathrm{SD}$. The Wilcoxon signed-rank test was used to evaluate the effects of each drug by compare values to the previous one. $P$-values less than 0.05 were considered significant. Statistical analysis was performed using SPSS version 21 (IBM Corp, Armonk, NY, USA).

\section{Results}

\section{Effects of Bisacodyl on the Phasic Contraction During Stimulation}

Phasic contractions were observed by EFS (Fig. 2). After ad-

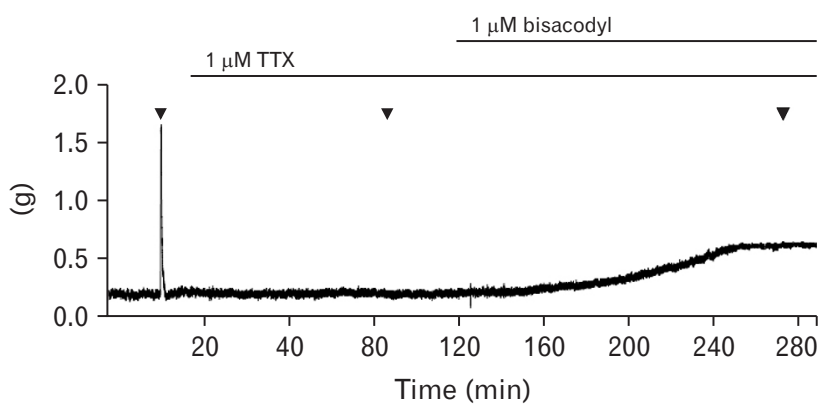

Figure 3. Typical trace of electrical field stimulation-induced responses of the human colon muscle strip after serial administration of tetrodotoxin (TTX) and bisacodyl in the circular muscle.

dition of atropine, EFS elicited early phasic relaxation and delayed contraction following cessation of EFS (Fig. 1). In longitudinal muscles ( $\mathrm{n}=13)$, the peak decreased from $1.55 \pm 0.72 \mathrm{~g}$ to $1.18 \pm$ $0.49 \mathrm{~g}$ after perfusion of bisacodyl (Fig. 4A). Peak values decreased further to $0.61 \pm 0.24 \mathrm{~g}$ after perfusion of atropine and increased to $0.71 \pm 0.20 \mathrm{~g}$ after addition of L-NNA. Subsequent addition of MRS2500 and TTX did not affect peak values. These observations indicate that bisacodyl decreases the peak contraction in the sigmoid colon longitudinal muscle, and cholinergic and nitrergic pathways are active in the peak contraction under bisacodyl perfusion. However, the nadir increased from $0.28 \pm 0.22 \mathrm{~g}$ to $0.82 \pm 0.25 \mathrm{~g}$ after perfusion of bisacodyl (Fig. 4B). Increased nadir persisted after sequential addition of atropine, L-NNA, MRS2500, and TTX, indicating that bisacodyl increases the tone in the sigmoid colon longitudinal muscle through direct action on the smooth muscle (Fig. 4B). On the other hand, bisacodyl did not show significant effects on the peak and nadir of the colonic circular muscle $(n=12)$ (Fig. $4 \mathrm{C}$ and $4 \mathrm{D})$.

\section{Effects of Bisacodyl on the Post-stimulus Rebound and Tone}

After cessation of EFS, PSR was produced (Fig. 2). Effects of bisacodyl on the PSR were similar to that during stimulation. In longitudinal muscles $(\mathrm{n}=13)$, the PSR peak decreased from $1.29 \pm$ $0.63 \mathrm{~g}$ to $0.60 \pm 0.58 \mathrm{~g}$ after perfusion of bisacodyl (Fig. $5 \mathrm{~A}$ ). The PSR peak decreased further to $0.08 \pm 0.54 \mathrm{~g}$, after perfusion of atropine and increased to $0.20 \pm 0.46 \mathrm{~g}$ after addition of L-NNA. Subsequent addition of MRS2500 and TTX did not affect the PSR peak. These observations indicate that bisacodyl decreases the PSR peak contraction in the sigmoid colon longitudinal muscle, and cholinergic and nitrergic pathways are active in the PSR peak contraction under bisacodyl perfusion. However, PSR tone in- 
A
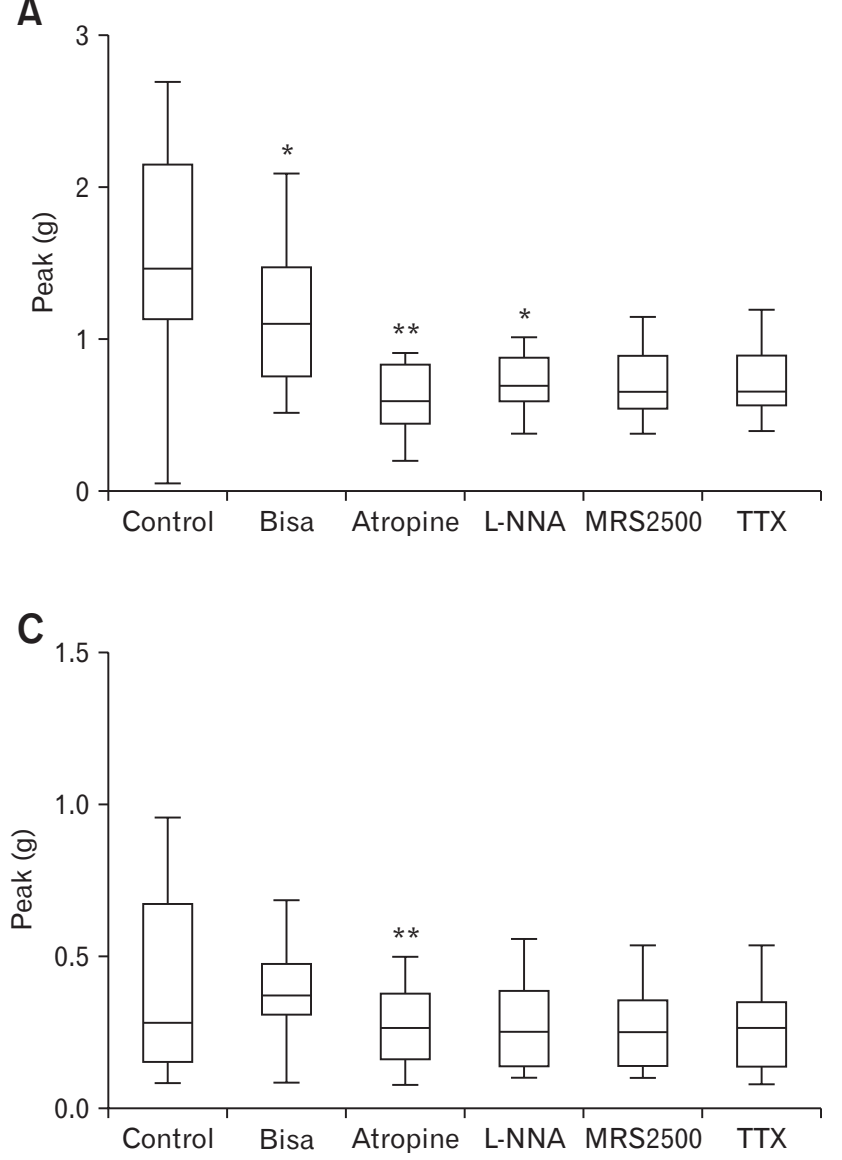

B

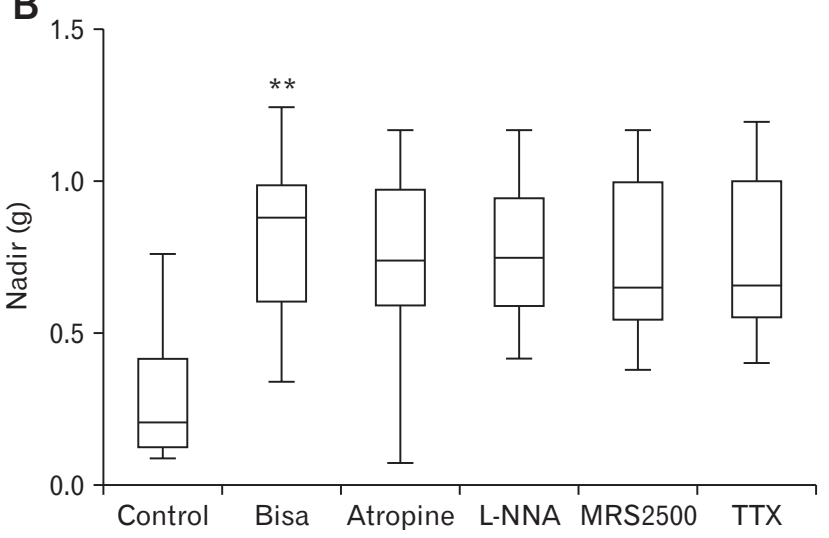

D

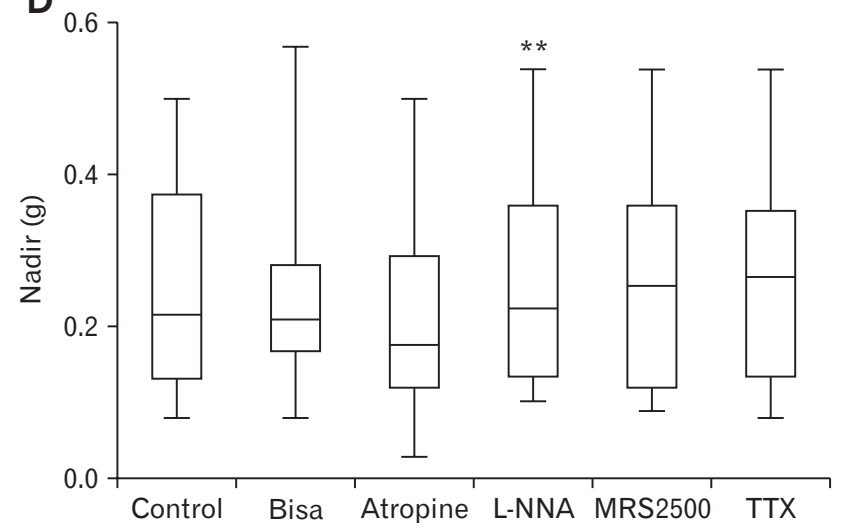

Figure 4. Peak and nadir during electrical field stimulation (EFS) after serial administration of bisacodyl, atropine, N-nitro-L-arginine (L-NNA), MRS2500, and tetrodotoxin (TTX). (A) Peak in the longitudinal muscle. (B) Nadir in the longitudinal muscle. (C) Peak in the circular muscle. (D) Nadir in the circular muscle. The Wilcoxon signed-rank test was used to evaluate the effects of each drug by compare values to the previous one: ${ }^{\star} P<0.05$ and ${ }^{*} P<0.01$.

creased from $0.41 \pm 0.54 \mathrm{~g}$ to $0.73 \pm 0.26 \mathrm{~g}$ after perfusion of bisacodyl (Fig. 5B). Increased PSR tone persisted after sequential addition of atropine, L-NNA, MRS2500, and TTX, indicating that bisacodyl increases the PSR tone in the sigmoid colon longitudinal muscle through direct action on the smooth muscle (Fig. 5B). Bisacodyl did not significant affect the post-stimulus peak and tone of the colonic circular muscle $(\mathrm{n}=12)$ (Fig. 5C and 5D).

\section{Effects of Bisacodyl on the Late Tone}

Late tone increased from $0.26 \pm 0.22 \mathrm{~g}$ to $0.80 \pm 0.24 \mathrm{~g}$ after perfusion of bisacodyl (Fig. 6A) in the longitudinal muscle ( $\mathrm{n}=$ 13), and from $0.18 \pm 0.09 \mathrm{~g}$ to $0.27 \pm 0.15 \mathrm{~g}$ (Fig. 6B) in the circular muscle $(n=12)$. Increased late tone persisted after sequential addition of atropine, L-NNA, MRS2500, and TTX, indicating that bisacodyl increases the late tone in the sigmoid colon longi- tudinal and circular muscles through direct action on the smooth muscle.

\section{Effects of Bisacodyl on the Longitudinal Muscle Under Pre-treatment of Tetrodotoxin}

Pre-treatment of TTX abolished EFS-induced phasic contraction (Fig. 3). No EFS-induced phasic contraction was produced after perfusion of bisacodyl. However, the tone increased from $0.140 \pm 0.141 \mathrm{~g}$ to $0.809 \pm 0.641 \mathrm{~g}$ after perfusion of bisacodyl in the longitudinal muscle $(\mathrm{n}=7)$, and from $0.167 \pm 0.074 \mathrm{~g}$ to $0.423 \pm 0.200 \mathrm{~g}$ in the circular muscle $(\mathrm{n}=6)$, indicating a direct mechanism of action of bisacodyl on the colon muscles.

The amount of increased tone and time latency to the maximal tone after perfusion of bisacodyl was compared between with and without pre-treatment of TTX. There were no differences in the 
A

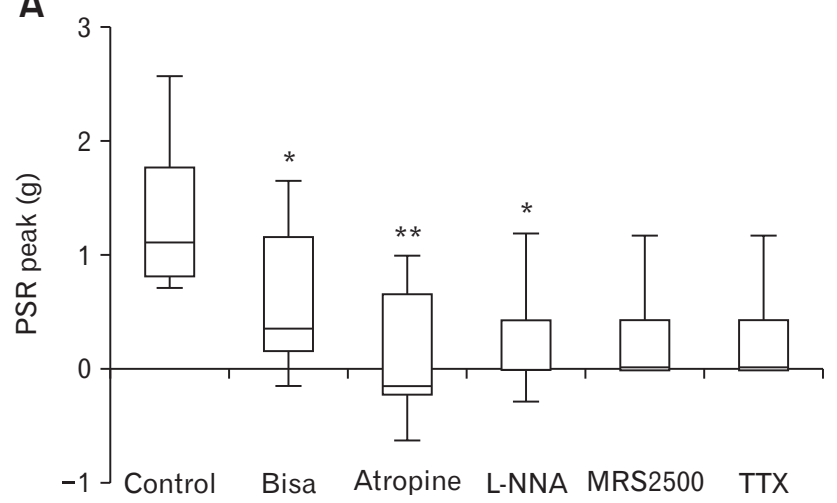

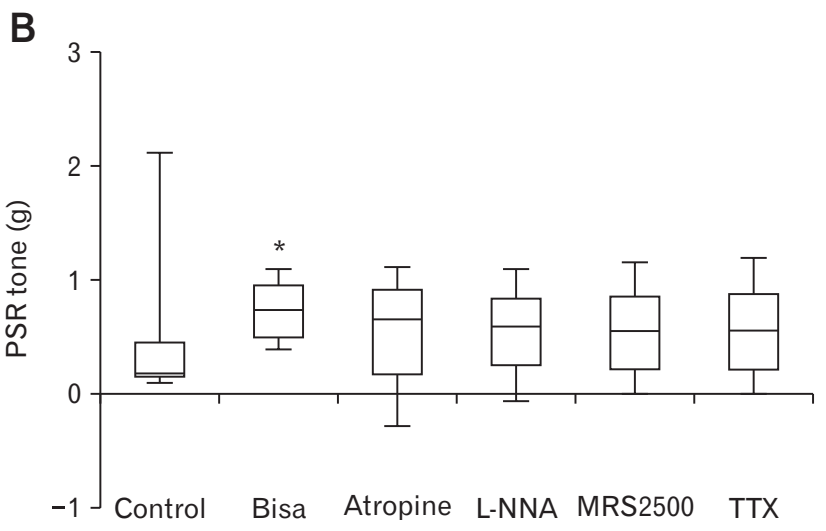

D

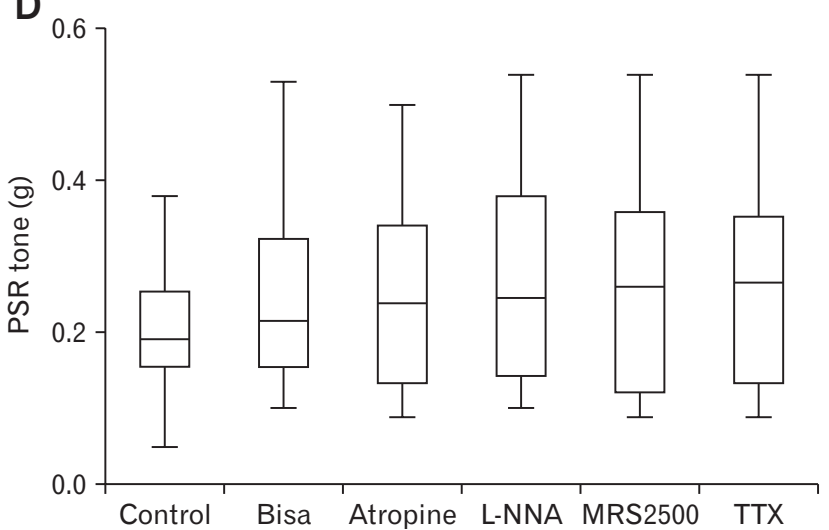

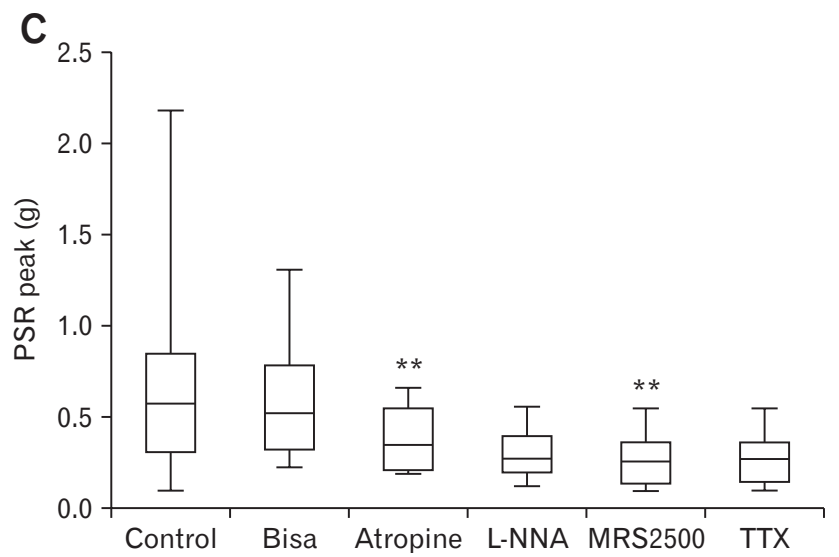

Figure 5. Post-stimulus rebound (PSR) peak and tone after serial administration of bisacodyl, atropine, N-nitro-L-arginine (L-NNA), MRS2500, and tetrodotoxin (TTX). (A) PSR peak in the longitudinal muscle. (B) PSR tone in the longitudinal muscle. (C) PSR peak in the circular muscle. (D) PSR tone in the circular muscle. The Wilcoxon signed-rank test was used to evaluate the effects of each drug by compare values to the previous one.: ${ }^{*} P<0.05$ and ${ }^{* *} P<0.01$.
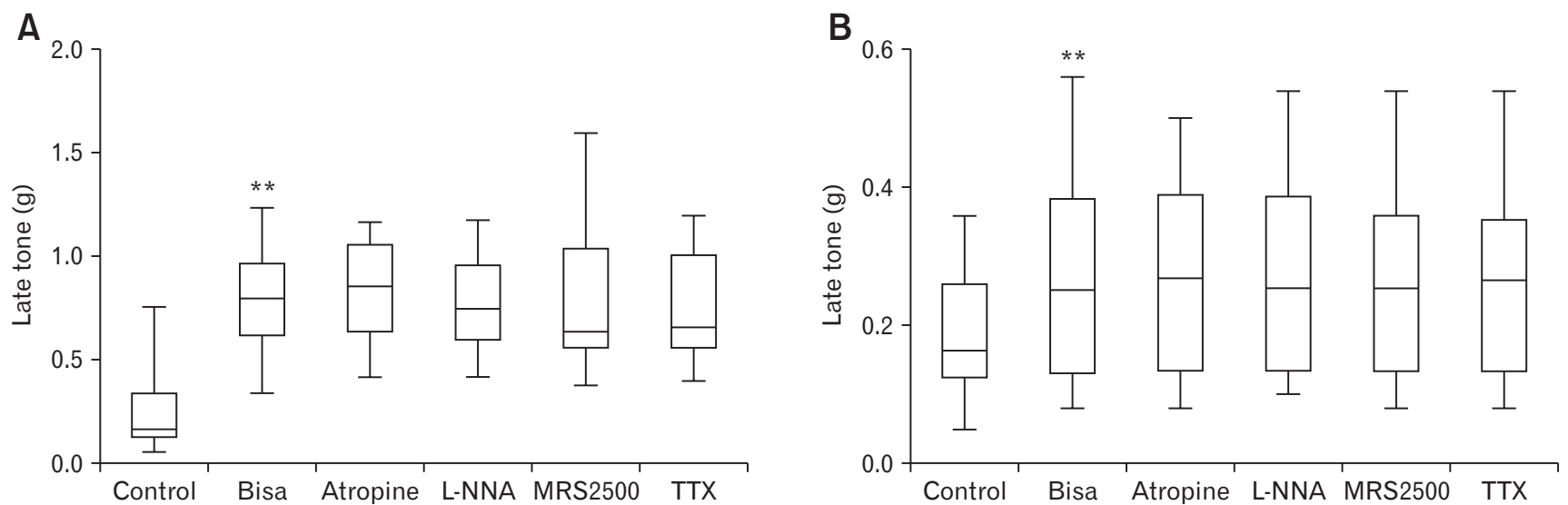

Figure 6. Late tone after serial administration of bisacodyl, atropine, N-nitro-L-arginine (L-NNA), MRS2500, and tetrodotoxin (TTX). (A) Late tone in the longitudinal muscle. (B) Late tone in the circular muscle. The Wilcoxon signed-rank test was used to evaluate the effects of each drug by compare values to the previous one.: ${ }^{*} P<0.05$ and ${ }^{*} P<0.01$. 
longitudinal muscle. Latency was longer with pre-treatment of TTX than without in the circular muscle (39.8 minutes vs 26.8 minutes), while there was no difference regarding the amount of increased tone.

\section{Discussion}

Bisacodyl is a potent stimulus laxative. Although bisacodyl has safety concerns relative to long-term use, it appears to be effective in practice. However, the underlying mechanism of action of bisacodyl on the colon remains to be elucidated, especially in humans. If the effects of bisacodyl on the human colon are well defined and its mechanism of action is elucidated, we could administer bisacodyl more effectively for patients with constipation. Thus, we investigated the effects of bisacodyl on human colon muscle contraction, using an organ bath technique, and elucidated its mechanism of action. Bisacodyl directly increases the tone of sigmoid colon muscles, while it decreases the peak contraction of the sigmoid longitudinal muscle, which probably is able to facilitate colonic propulsion.

In an early German study, bisacodyl increased the tone of isolated muscle strips of the human colon. ${ }^{18}$ The effect of bisacodyl could not be prevented by TTX, and abolished by pre-treatment with verapamil, antagonizing calcium influx into the muscle cell, showing similar results to the current study. On the contrary, an increase in the sporadic spiking activity during stimulation by bisacodyl has been reported in humans. ${ }^{5}$ This electromechanical coupling vanishes with the application of Lidocaine onto the colon mucosa indicating that bisacodyl acts on the mucosal nerve endings. ${ }^{19}$ An experimental animal study revealed that bisacodyl inhibits water absorption by increasing the secretion of prostaglandin $\mathrm{E}_{2}$ from macrophages in the colon mucosa. ${ }^{20}$ However, prostaglandin $\mathrm{E}_{2}$ induces longitudinal smooth muscle contraction via the $\mathrm{EP}_{1}$ receptor in the human colon. ${ }^{21}$ Thus, the direct effect on the colon smooth muscle, and an activation of mucosal endings of myenteric and submucosal neurons have been suggested as a mechanism of action of bisacodyl. $^{22}$

Colonic HAPC is an important mechanism for facilitating the transit of colonic contents over long distances, and often precedes defecation. ${ }^{22,23}$ Intraluminal infusion of bisacodyl can elicit HAPC in humans. ${ }^{5,19}$ Thus, bisacodyl is administered for a pharmacologic provocation to assess colonic neuromuscular function integrity. ${ }^{3,4}$ In a recent study, a higher dose of bisacodyl during colonic manometry increased the number of propagated HAPCs. ${ }^{24}$ This implies that an activation of mucosal nerve endings may contribute more to the mechanism of action of bisacodyl, compared to a direct effect on the colon smooth muscle, because the intrinsic neural reflex within the colonic myenteric plexus has been suggested as a potential underlying mechanism of HAPC. ${ }^{25}$ However, the tonic contraction of the longitudinal muscle by a direct effect of bisacodyl on the smooth muscle could reduce colon elongation, which improves the spatiotemporal-coordinated pattern of HAPCs. Thus, bisacodyl would improve HAPCs in terms of facilitating the transit of colon contents. From the practical point of view, bisacodyl could be effectively administered for managing constipation in patients with neuropathy.

In the present study, it took about 60 minutes to reach the maximal response (increased basal tone of colon muscle strips) of bisacodyl. Interestingly, the potentiated tone lasted more than 2 hours (Fig. 1). The effect of bisacodyl gradually reduced and nearly abolished at 2 hours by washout (data not shown). Although it is uncertain how much the direct effect of bisacodyl is involved in practical use, we could expect its persistent direct effect on the colon when the concentration of biascodyl is maintained.

In the present study, the peak EFS-induced phasic contraction was decreased by perfusion of bisacodyl during and after cessation of EFS only in the longitudinal muscle. Although it is difficult to define the role of decreased peak contraction presently, that might contribute to effective colon transit. Contrary to the tone, phasic contractions with a high peak are not thought to be helpful for propelling colonic contents. However, the effect of peak contraction on the colon transit could be different according to the colonic location. Therefore, identical experiments using the proximal colon muscle strips are required. In addition, further experiments with a different methodology are warranted to investigate the underlying mechanism of decreased peak contraction by bisacodyl.

In conclusion, the present study provides evidence that a direct action of bisacodyl on the smooth muscle increases the tone of longitudinal muscle in the human sigmoid colon, suggesting that bisacodyl could be effectively administered for managing constipation in patients with neuropathy. Further study is warranted to investigate the neural mechanism of action of bisacodyl.

\section{Financial support: None.}

\section{Conflicts of interest: None.}

Author contributions: Yang Won Min contributed to the data analysis and interpretation, and drafted the manuscript; Eun-ju Ko and Ji Yeon Lee performed experiments; Jeong Hwan Kim contributed to the data interpretation and edited the manuscript; Hee 
Cheol Kim and Woo Yong Lee contributed to the data collection; Poong-Lyul Rhee designed and coordinated the study, contributed to the data interpretation, and edited the manuscript; and all the authors approved the final version of the manuscript.

\section{References}

1. Shin JE, Jung HK, Lee TH, et al. Guidelines for the diagnosis and treatment of chronic functional constipation in Korea, 2015 revised edition. J Neurogastroenterol Motil 2016;22:383-411.

2. Adams WJ, Meagher AP, Lubowski DZ, King DW. Bisacodyl reduces the volume of polyethylene glycol solution required for bowel preparation. Dis Colon Rectum 1994;37:229-233; discussion 233-224.

3. Hamid SA, Di Lorenzo C, Reddy SN, Flores AF, Hyman PE. Bisacodyl and high-amplitude-propagating colonic contractions in children. J Pediatr Gastroenterol Nutr 1998;27:398-402.

4. Bassotti G, Chiarioni G, Germani U, Battaglia E, Vantini I, Morelli A. Endoluminal instillation of bisacodyl in patients with severe (slow transit type) constipation is useful to test residual colonic propulsive activity. Digestion 1999;60:69-73.

5. Schang JC, Hemond M, Hebert M, Pilote M. Changes in colonic myoelectric spiking activity during stimulation by bisacodyl. Can J Physiol Pharmacol 1986;64:39-43.

6. American Gastroenterological Association, Bharucha AE, Dorn SD, Lembo A, Pressman A. American Gastroenterological Association medical position statement on constipation. Gastroenterology 2013;144:211217.

7. Müller-Lissner SA, Kamm MA, Scarpignato C, Wald A. Myths and misconceptions about chronic constipation. Am J Gastroenterol 2005;100:232-242.

8. Xing JH, Soffer EE. Adverse effects of laxatives. Dis Colon Rectum 2001;44:1201-1209.

9. Sajid MS, Hebbar M, Baig MK, Li A, Philipose Z. Use of prucalopride for chronic constipation: a systematic review and meta-analysis of published randomized, controlled trials. J Neurogastroenterol Motil 2016;22:412-422.

10. Eswaran S, Guentner A, Chey WD. Emerging pharmacologic therapies for constipation-predominant irritable bowel syndrome and chronic constipation. J Neurogastroenterol Motil 2014;20:141-151.

11. Jarmuz A, Zielinska M, Storr M, Fichna J. Emerging treatments in Neurogastroenterology: perspectives of guanylyl cyclase $\mathrm{C}$ agonists use in functional gastrointestinal disorders and inflammatory bowel diseases. Neurogastroenterol Motil 2015;27:1057-1068.
12. Miner PB, Jr., Camilleri M, Burton D, et al. Prucalopride induces high-amplitude propagating contractions in the colon of patients with chronic constipation: a randomized study. Neurogastroenterol Motil 2016;28:1341-1348.

13. Min YW, Hong YS, Ko EJ, et al. Impairment of the proximal to distal tonic gradient in the human diabetic stomach. Neurogastroenterol Motil 2014;26:229-236.

14. Min YW, Hong YS, Ko EJ, et al. Nitrergic pathway is the main contributing mechanism in the human gastric fundus relaxation: an in vitro study. PLoS One 2016;11:e0162146.

15. Sinn DH, Min BH, Ko EJ, et al. Regional differences of the effects of acetylcholine in the human gastric circular muscle. Am J Physiol Gastrointest Liver Physiol 2010;299:G1198-G1203.

16. Min YW, Ko EJ, Lee JY, et al. Nitrergic pathway is the major mechanism for the effect of DA-9701 on the rat gastric fundus relaxation. J Neurogastroenterol Motil 2014;20:318-325.

17. Franck H, Kong ID, Shuttleworth CW, Sanders KM. Rebound excitation and alternating slow wave patterns depend upon eicosanoid production in canine proximal colon. J Physiol 1999;520(Pt 3):885-895.

18. Mitznegg P, Schubert E, Domschke W, et al. [Mode of action of bisacodyl (dulcolax) on isolated muscles of human colon (author's transl).] Klin Wochenschr 1975;53:493-495. [German]

19. Preston DM, Lennard-Jones JE. Pelvic motility and response to intraluminal bisacodyl in slow-transit constipation. Dig Dis Sci 1985;30:289294.

20. Ikarashi N, Baba K, Ushiki T, et al. The laxative effect of bisacodyl is attributable to decreased aquaporin- 3 expression in the colon induced by increased PGE2 secretion from macrophages. Am J Physiol Gastrointest Liver Physiol 2011;301:G887-G895.

21. Fairbrother SE, Smith JE, Borman RA, Cox HM. Characterization of the EP receptor types that mediate longitudinal smooth muscle contraction of human colon, mouse colon and mouse ileum. Neurogastroenterol Motil 2011;23:782-e336.

22. Bharucha AE. High amplitude propagated contractions. Neurogastroenterol Motil 2012;24:977-982.

23. Cook IJ, Furukawa Y, Panagopoulos V, Collins PJ, Dent J. Relationships between spatial patterns of colonic pressure and individual movements of content. Am J Physiol Gastrointest Liver Physiol 2000;278:G329-G341.

24. Borrelli O, Pescarin M, Saliakellis E, et al. Sequential incremental doses of bisacodyl increase the diagnostic accuracy of colonic manometry. Neurogastroenterol Motil 2016;28:1747-1755.

25. Dinning PG, Zarate N, Hunt LM, et al. Pancolonic spatiotemporal mapping reveals regional deficiencies in, and disorganization of colonic propagating pressure waves in severe constipation. Neurogastroenterol Motil 2010;22:e340-e349 